\title{
Distribution of HLA-B*27 Subtypes in Patients with Ankylosing Spondylitis in Local Population
}

\author{
Noor Afshan Lodhi, Muhammad Mukarram Bashir, Hamid Nawaz Tipu, and Muhammad Hussain
}

\begin{abstract}
Objective: To compare the distribution of HLA-B ${ }^{\star} 27$ subtypes in healthy controls and in ankylosing spondylitis (AS) patients of different ethnic groups from Pakistan.

Study Design: Descriptive study.

Place and Duration of Study: Armed Forces Institute of Pathology, Rawalpindi, Pakistan, from April 2016 to October 2017.

Methodology: Forty-nine HLA-B*27 positive, unrelated AS patients and 18 HLA-B*27 positive healthy BMT/renal transplant donors were selected for this study. Typing of the HLA-B27 alleles was performed by the polymerase chain reaction-sequence-specific primer (PCR-SSP).

Results: There was a wide number of HLA-B 27 subtypes and an elevated frequency of the $B^{*} 2707$ allele in the AS patients. The allele $B^{\star} 2706$ seems to have a protective role in the population studied because it was found only in the healthy controls. HLA-B*27:03 and 07 were found predominant subtypes in Punjabis and Pathans, respectively.

Conclusion: There were no significant differences for the distribution of $B^{*} 27$ subtypes between patients and controls $(p>0.05)$.
\end{abstract}

Key Words: Autoimmunity, Ankylosing spondylitis, Human leukocyte antigen, Alleles, Polymorphism, Ethnic groups, PCR-SSP.

\section{INTRODUCTION}

Ankylosing spondylitis (AS) is chronic disabling disease characterised by inflammation of the axial skeleton, as well as extra spinal involvement. Etiology of this immune-mediated disease is not fully understood but genetic factors play a key role in AS susceptibility. Class I Human Leukocyte Antigen, (HLA)-B*27, is strongly associated with AS. This antigen is found in over $95 \%$ of the patients of AS in the Caucasian population. 1 Arthritogenic peptide model proposes that HLA-B ${ }^{*} 27$ acts as restriction molecule for antigenic peptides, which might be derived from bacterial proteins or structurally related self-peptides, which would be presented to and cross-recognised by cytotoxic CD8+ $T$ lymphocytes. Supporting this theory, HLA-B*27 restricted CD8+ CTL clones with specificity for bacteria or autoantigens were detected in the synovial fluid and the peripheral blood of patients with AS. The HLA-B*27 alleles are discriminated in susceptible alleles and protective alleles, on the basis of their increased binding affinity of arthritogenic peptides or reduced binding affinity. ${ }^{2}$ These subtypes differ only in a few amino acids lining the peptide-binding cleft. Different HLA-B*27 alleles affect the biochemical

Department of Immunology, Armed Forces Institute of Pathology (AFIP), Rawalpindi, Pakistan

Correspondence: Dr. Noor Afshan Lodhi, Department of Immunology, Armed Forces Institute of Pathology (AFIP),

Harley Street, 46000, Rawalpindi, Pakistan

E-mail:afshan_ns@yahoo.com

Received: July 02, 2018; Accepted: November 30, 2018 and three-dimensional (3D) structure as well as the charge of the peptide-binding groove of the alpha chain. ${ }^{1,3}$

Different HLA-B*27 alleles may be associated with different ethnic groups, clinical manifestations, the age of onset, and prognosis. 4 The susceptible alleles for AS in different geographic locations of the globe are different. Iran, Turkey, and Syria have $B^{\star} 2705$ and $B^{\star} 2702$, China and Japan have $B^{*} 2704$ and $B^{*} 2705$, Malaysia has $B^{\star} 2706$ and $B^{\star} 2704$, Korea and India have $B^{\star} 2705$ and $B^{*} 2704$, while Lebanese, Semitic and Greek Cypriot populations have predominant $B^{*} 2702$. An ancestor HLA-B*27 allele, i.e. $B^{*} 2705$, is present in almost all populations. ${ }^{5,6}$

Although HLA-B*27 alleles studies have not been conducted, but HLA frequency studies have been conducted earlier in Pakistan. In a study conducted at the Aga Khan University, Moatter et al. reported an overall prevalence of HLA-B ${ }^{\star} 27$ in Pakistan of $1.3 \%$, without mentioning ethnicities. ${ }^{7}$ Mohyuddin et al. concluded that the Pathan and Kalash have a relatively higher frequency of $B^{*} 27$ than the rest of the population, $9.4 \%$ and $4.2 \%$ respectively. Punjabis were not included in this study. ${ }^{8}$ Another study by Ali Raza et al., reported the frequency of HLA-B ${ }^{\star} 27$ in the Gujjar (Punjabis) as $2.1 \% .{ }^{9}$

The purpose of this study was to investigate the association of HLA-B*27 allele subtypes with AS patients, which has never been studied before in Pakistan using the polymerase chain reaction-sequence-specific primer (PCR-SSP) and to determine the distribution of these polymorphisms in different ethnic groups. 


\section{METHODOLOGY}

During the period of April 2016 to October 2017, 517 symptomatic individuals were screened for HLA-B 27 antigen by flow cytometry at the Armed Forces Institute of Pathology, Rawalpindi. Forty-nine HLA-B ${ }^{\star} 27$ positive, unrelated individuals, fulfilling modified New York criteria were included in this study. ${ }^{10}$ Individuals who had history of the low back pain and stiffness of less than 3 months' duration and demonstrated no limitation of lumber spine movement were excluded. Participation was entirely voluntary; patients were briefed about the study and their informed written consent was obtained. Institution Review Board had given approval for the study.

The control group consisted of $18 \mathrm{HLA}^{*}{ }^{*} 27$ positive (PCR-SSP kit method) healthy subjects (mean age 15.33 years \pm 11.59 ) on the list of transplant donors (prospective donors), with no history of back pain and comorbidity. These participants had the same sociodemographic characteristics as the AS population.

Venous blood was collected from patients and controls, DNA was isolated using the Gentra Puregene Blood kit by Qiagen, Minnesota, USA, according to the manufacturer's instructions. DNA was stored at $-20^{\circ} \mathrm{C}$ until analysis. The alleles of HLA-B ${ }^{*} 27$ were typed by using the PCR-SSP designed by Downing et al.,11 which allowed the identification of HLA-B*2701 through $B^{\star} 2730$ alleles.
Statistical analyses were performed using SPSS software (version SPSS 24.0). The proportion of $B^{*} 27$ alleles were compared in cases and control, using Fisher exact test ( $R$ program). The differences between the results were considered significant at $p<0.05$.

\section{RESULTS}

As shown in Table I, the majority of $B^{*} 27$ positive AS patients were young males (45 men, 4 women), mean age $32.57 \pm 9.0$ years in this study. The distribution of $B^{*} 27$ alleles in patient with $A S$ and healthy controls is given in Table II. HLA-B*27:07 was the most frequent allele in cases while $B^{*} 27: 06$ was found in the control group only. Five alleles were common in AS patients and in controls $\left(B^{\star} 27: 03,04,05,07\right.$ and $\left.B^{*} 27: 28\right)$. $B^{\star} 2705$

Table I: Basic demographics of the ankylosing spondylitis patient included in this study.

\begin{tabular}{lc}
\hline Demographic value & $\begin{array}{c}\text { AS patients (New York criteria) } \\
\mathrm{n}=49(\%)\end{array}$ \\
\hline Male (\%) & $45(91.8 \%)$ \\
Female (\%) & $4(8.16 \%)$ \\
Mean age (years) & $32.57 \pm 9.0$ \\
Ethnicity & \\
Panjabi & $26(53.1 \%)$ \\
Pathan & $11(22.4 \%)$ \\
Saraiki & $4(8.2 \%)$ \\
Kashmiri / Gilgiti & $5(10.2 \%)$ \\
Others & $3(6 \%)$ \\
\hline
\end{tabular}

${ }^{*}=$ Hindko 1, Baloch 1, Urdu speaking 1.

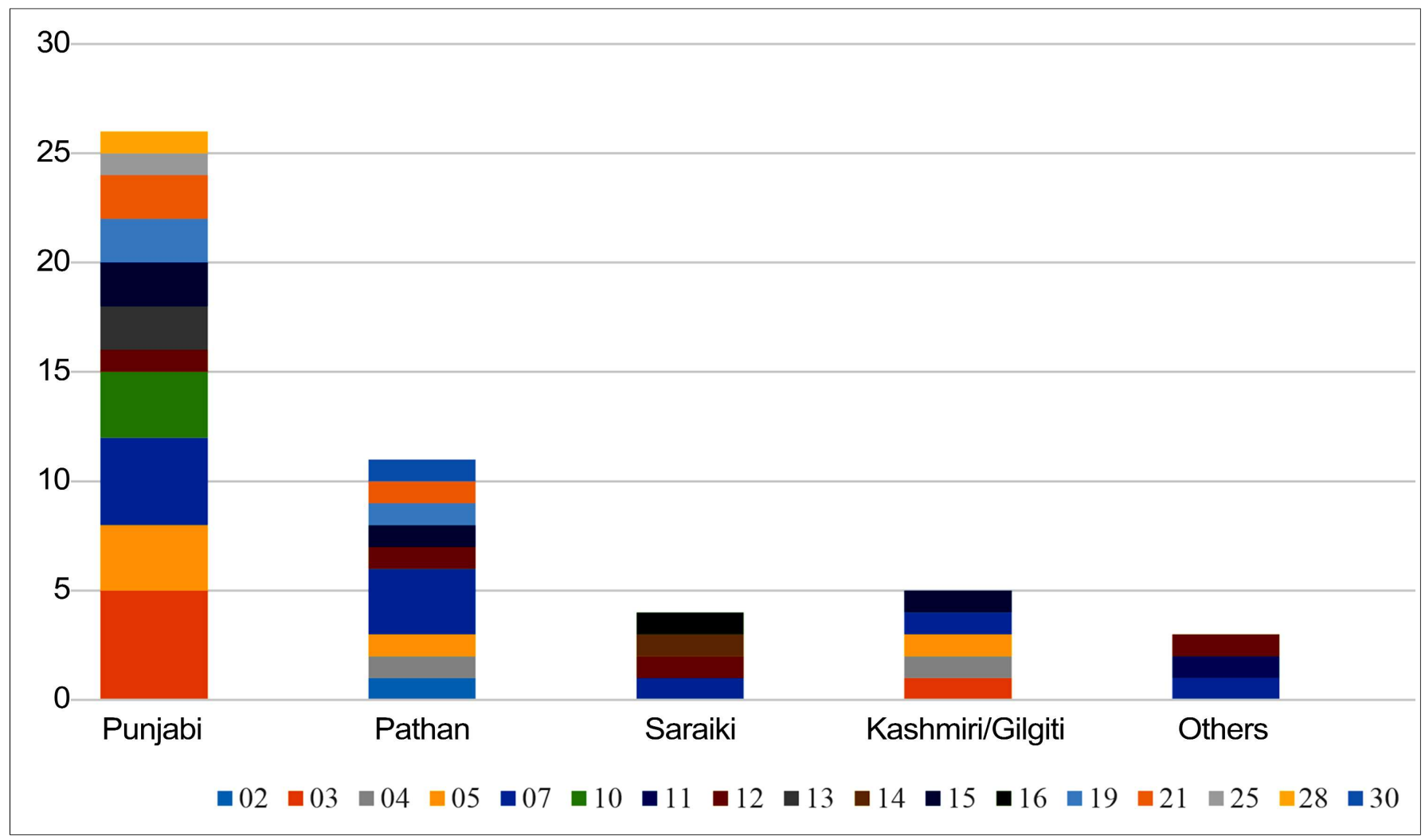

Figure 1: Distribution of HLA-B`27 subtypes in different ethnic groups of Pakistan. Total 26 (53.1\%) Punjabis and 11 (22.4\%) Pathan AS patients were depicted here. Punjabis have predominant HLA-B ${ }^{\star} 27: 03$, while Pathans have HLA-B`27:07, Saraiki 4 (8.2\%), Kashmiri/Gilgiti 5 (10.2\%), whereas, "others" include Urdu speaking, Hindko and Baloch, one patient each group ( $2 \%$ each). 
Table II: The distribution of $B^{\star} 27$ alleles in patients with AS and healthy controls.

\begin{tabular}{l|l|l|l}
\hline HLA-B*27 alleles & $\begin{array}{c}\text { AS patients } \\
\mathrm{n}=49(\%)\end{array}$ & $\begin{array}{c}\text { Controls } \\
\mathrm{n}=18(\%)\end{array}$ & p-value \\
\hline 02 & $1(2)$ & 0 & 0.59 \\
03 & $6(12.2)$ & $3(16.7)$ & 0.94 \\
04 & $2(4.1)$ & $3(16.7)$ & 0.22 \\
05 & $5(10.2)$ & $5(27.8)$ & 0.16 \\
06 & 0 & $2(11.1)$ & 0.11 \\
07 & $10(20.4)$ & $4(22.2)$ & 0.85 \\
10 & $3(6.1)$ & 0 & 0.68 \\
11 & $1(2)$ & 0 & 0.59 \\
12 & $4(8.2)$ & 0 & 0.50 \\
13 & $2(4.1)$ & 0 & 0.95 \\
14 & $1(2)$ & 0 & 0.59 \\
15 & $4(8.2)$ & 0 & 0.50 \\
16 & $1(2)$ & 0 & 0.59 \\
19 & $3(6.1)$ & 0 & 0.68 \\
21 & $3(6.1)$ & 0 & 0.68 \\
25 & $1(2)$ & 0 & 0.59 \\
28 & $1(2)$ & $1(5.6)$ & 0.95 \\
30 & $1(2)$ & 0 & 0.59 \\
\hline
\end{tabular}

and $B^{*} 2707$ were predominant alleles in both the AS and controls.

Frequencies of the HLA-B 27 alleles between the patients and the control were compared and tested separately for each subtype, using the Fisher's exact test. However, there was no significant difference between the patients and healthy individuals $(p>0.11)$. Among different ethnic groups, in Punjabi $B^{*} 27: 03,07$ and 10 were $19.2 \%(n=5), 15.4 \%(n=4)$ and $11.5 \%(n=3)$, respectively; and Pathan had predominant $B * 27: 07$ $(n=3,27.3 \%) . B^{\star} 27: 07,12,14$ and $B^{\star} 27: 16$ was found in 4 Saraiki patients, while $B^{\star} 27: 03,04,05,07$ and $B^{*} 27: 15$ was found in 5 Kashmiri/Gilgitis (Figure 1).

\section{DISCUSSION}

HLA-B*27 has 106 alleles; whereas, different subtypes show population specific distribution. Some of these subtypes confer the risk of AS while others are protective. Chinese and northern Indians have susceptible allele, predominantly HLA-B*27:04, HLA-B*27:05; West India reported HLA-B*27:07 as a major susceptible allele in addition to above-mentioned alleles. ${ }^{12}$ In Iran and Turkey, HLA-B*27:02 and HLA-B*27:05 are common AS associated alleles. 6,13

HLA-B*27:07 was found as the predominant allele in AS patients in current study, followed by HLA-B*27:03 and HLA-B ${ }^{\star} 27: 05$. These findings are supported by other studies conducted globally. ${ }^{14}$ However, GarciaFernandez reported $B^{*} 2707$ is present in a higher frequency in the Jewish population (12.5\%) compared to Asian Indians (5.7\%) and Chinese (2\%). ${ }^{15}$ VarnavidouNicolaidou et al. reported a protective role of $B^{*} 27: 07$ against AS. ${ }^{16}$ The discrepancy may be a demonstration of the difference in the sample sizes, the geographical variation, and the ethnic background. HLA-B*2705 is an ancestor allele found in both AS and control group, with widespread distribution from East Asia (China 11.3\%) to South Asia (Pakistan 10.2\% this study, India 18\%, Iran $48.4 \%$ ) to West Asia (Turkey $37.8 \%$ ). ${ }^{12}$

In addition to two subtypes mentioned above, $B^{*} 27: 02$, $03,04,10,12,13,15,19$ and $B^{\star} 27: 21$ were reported in this study in AS patients but there were no significant differences for the distribution of $B^{\star} 27$ subtypes between patients and controls $(p>0.05)$. $B^{\star} 2706$ was observed in control group only, several studies have reported that HLA-B*2706 is not associated with AS and may protect against the development of AS. ${ }^{17}$

Pathan (Pashtuns) and Kalash have the relatively higher frequency of $B^{*} 27$ than other ethnic groups. ${ }^{8}$ Interestingly, we found Punjabis to have a higher frequency of $B^{\star} 27$ as the majority of considered patients were Punjabis. This discrepancy might be due to the location of this Institute and smaller sample size. A larger sample size studies may be required to clarify this disparity.

It is expected that classification criteria for many diseases will be modified on the basis of their genetic makeup. More genetic studies in various ethnic groups will contribute to the already existing findings of the disease's negative or positive association of the various HLA-B27 subtypes and possibly other genes. The outcome of such studies will be a better understanding of the possible mechanisms involved in these associations.

\section{CONCLUSION}

The current study demonstrated the distribution of HLA$B^{\star} 27$ in different ethnic and geographic populations of Pakistan. HLA-B*27:03 and 07 was predominant subtype in Punjabis and Pathans, respectively; while HLA-B*27:06 was found in control group only. There were no significant differences for the distribution of $B^{\star} 27$ subtypes between patients and controls $(p>0.05)$.

\section{REFERENCES}

1. Bowness P. HLA-B27. Annu Rev Immunol 2015; 33:29-48.

2. Schittenhelm RB, Sivaneswaran S, Lim Kam Sian TC, Croft NP, Purcell AW. HLA-B27 allotype-specific binding and candidate arthritogenic peptides revealed through heuristic clustering of DIA-MS data. Mol Cell Proteomics 2016; 15:1867-76.

3. Winkler K, Winter A, Rueckert C, Uchanska-Ziegler B, Alexiev U. Natural MHC class I polymorphism controls the pathway of peptide dissociation from HLA-B27 complexes. Biophys $J$ 2007; 93:2743-55.

4. Mou Y, Zhang P, Li Q, Lin Z, Liao Z, Wei Q, et al. Clinical features in juvenile-onset ankylosing spondylitis patients carrying different B27 subtypes. Biomed Res Int 2015; 2015: 594878.

5. Fallahi S, Mahmoudi M, Nicknam MH, Gharibdoost F, Farhadi E, Saei $A$, et al. Effect of HLA-B*27 and its subtypes on clinical 
manifestations and severity of ankylosing spondylitis in Iranian patients. Iran J Allergy Asthma Immunol 2013; 12:321-30.

6. Lin $\mathrm{H}$, Gong $\mathrm{YZ}$. Association of HLA-B27 with ankylosing spondylitis and clinical features of the HLA-B27-associated ankylosing spondylitis: A meta-analysis. Rheumatol Int 2017; 37:1267-80

7. Moatter T, Aban M, Tabassum S, Shaikh U, Pervez S. Molecular analysis of human leukocyte antigen class I and class II allele frequencies and haplotype distribution in Pakistani population. Indian J Hum Genet 2010; 16:149-54.

8. Mohyuddin A, Ayub Q, Khaliq S, Mansoor A, Mazhar K, Rehman S, et al. HLA polymorphism in six ethnic groups from Pakistan. Tissue Antigens 2002; 59:492-501.

9. Raza A, Firasat S, Khaliq S, Abid A, Shah SS, Mehdi SQ, et al. HLA class I and II polymorphisms in the Gujjar population from Pakistan. Immunol Invest 2013; 42:691-700.

10. Raychaudhuri SP, Deodhar A. The classification and diagnostic criteria of ankylosing spondylitis. J Autoimmun 2014; 49:128-33.

11. Downing J, Coates E, Street J, Hammond L, Rees TJ, Pepperall J, et al. A high-resolution polymerase chain reactionsequence-specific primer HLA B27 typing set and its application in routine HLA B27 testing. Genet Test 2006; 10:98-103.
12. Haridas V, Shetty P, Kumar MN, Vasanthakumar KC, Haridas K, Khode $\mathrm{V}$, et al. Human leukocyte antigen-B*27 allele subtype prevalence and disease association of ankylosing spondylitis among South Indian population. Indian J Rheumatol 2018; 13:38-43.

13. Diyarbakir E, Eyerci N, Melikoglu M, Topcu A, Pirim I. HLA B27 subtype distribution among patients with ankylosing spondylitis in eastern Turkey. Genet Test Mol Biomarkers 2012; 16:456-8.

14. Robinson PC, Brown MA. The genetics of ankylosing spondylitis and axial spondyloarthritis. Rheum Dis Clin North Am 2012; 38:539-53.

15. García-Fernández S, Gonzalez S, Miña Blanco A, MartinezBorra J, Blanco-Gelaz M, López-Vazquez A, et al. New insights regarding HLA-B27 diversity in the Asian population. Tissue Antigens 2001; 58:259-62.

16. Varnavidou-Nicolaidou A, Karpasitou K, Georgiou D, Stylianou G, Kokkofitou A, Michalis C, et al. HLA-B27 in the Greek Cypriot population: Distribution of subtypes in patients with ankylosing spondylitis and other HLA-B27-related diseases. The possible protective role of $\mathrm{B}^{\star} 2707$. Hum Immunol 2004; 65:1451-4.

17. Alexander F. Does HLA-B ${ }^{\star} 2706$ protect against ankylosing spondylitis? A meta-analysis. Int J Rheum Dis 2012; 15:8-12. 